\title{
The mechanochemically initiated Achmatowicz rearrangement
}

Carolin Falenczyk, Benjamin Pölloth, Petra Hilgers and Burkhard König* 


\title{
Supporting Information
}

\author{
Table of Contents
}

Page

S3

S4

\section{Contents}

Synthesis

General preliminary remark

Experimental details and ${ }^{1} \mathrm{H}-\mathrm{NMR}$ data for compounds $2 \mathrm{c}$,e and $3 \mathrm{~b}-\mathrm{d}$ Supplementary References 


\section{Experimental}

\section{General}

Starting materials were purchased from commercial suppliers and used without any further purification. Solvents were used in p.a. quality and dried according to common procedures, if necessary. The mechanochemical reactions were performed on a Fritsch Pulverisette-6 (repulsion motor: $180 \mathrm{~W}$, operating frequency: $200-425 \mathrm{rpm}$ ). The volume of the agate beaker was $500 \mathrm{~cm}^{3}$ containing ten agate milling balls $(8-10 \mathrm{~mm})$. Thin-layer chromatography (TLC) for reaction monitoring was performed with alumina plates coated with Merck silica gel $60 \mathrm{~F}_{254}$ (layer thickness: $0.2 \mathrm{~mm}$ ) and analyzed under UV-light $(254 \mathrm{~nm})$. Flash column chromatography was performed with Sigma Aldrich MN silica gel 60M (0.040-0.063 mm, 230-400 mesh) as stationary phase. For lyophilization a Christ ALPHA 1-4 was used. NMR spectra were recorded using a Bruker Avance $300\left({ }^{1} \mathrm{H}\right.$ : $\left.300 \mathrm{MHz}, \mathrm{T}=295 \mathrm{~K}\right)$ or a Bruker Avance $400\left({ }^{1} \mathrm{H}: 400 \mathrm{MHz},{ }^{13} \mathrm{C}\right.$ : $101 \mathrm{MHz}, \mathrm{T}=300 \mathrm{~K}$ ) instrument. The spectra are referenced against the NMR solvent and are reported as follows: ${ }^{1} \mathrm{H}$ : chemical shift $\delta(\mathrm{ppm})$, multiplicity $(\mathrm{s}=$ singlet, $\mathrm{d}=$ doublet, $\mathrm{t}=$ triplet, $\mathrm{q}$ $=$ quartet, $\mathrm{dd}=$ doublet of doublet, $\mathrm{qd}=$ quartet of doublet $)$, integration, coupling constant $(J$ in $\mathrm{Hz})$.

\section{Synthesis}

Ethyl 3-(furan-2-yl)-3-hydroxypropanoate (2c): ${ }^{[1]}$ The agate beaker containing ten agate milling balls was filled with $\mathrm{Zn}(1.0 \mathrm{~g}), \mathrm{NH}_{4} \mathrm{Cl}(0.40 \mathrm{~g}), \mathbf{1 a}(83 \mu \mathrm{L}, 1.0 \mathrm{mmol})$ and $4(310 \mu \mathrm{L}$, $3.0 \mathrm{mmol}$ ) and milling was performed for $45 \mathrm{~min}$. The reaction mixture was diluted with $\mathrm{Et}_{2} \mathrm{O}$ $(10 \mathrm{~mL})$ and a saturated solution of $\mathrm{NH}_{4} \mathrm{Cl}(10 \mathrm{~mL})$ and filtrated. The aqueous phase was extracted with $\mathrm{Et}_{2} \mathrm{O}(3 \times 15 \mathrm{~mL})$. The combined organic phases were washed with water $(50 \mathrm{~mL})$ and dried over $\mathrm{MgSO}_{4}$. The solvent was removed under reduced pressure and $2 \mathrm{c}(70 \mathrm{mg}, 38 \%)$ was obtained as brown oil. ${ }^{1} \mathrm{H}-\mathrm{NMR}\left(400 \mathrm{MHz}, \mathrm{CDCl}_{3}\right): \delta=7.33(\mathrm{~d}, 1 \mathrm{H}, J=1.8 \mathrm{~Hz}), 6.29$ (dd, $1 \mathrm{H}, J=1.8,3.2 \mathrm{~Hz}), 6.23(\mathrm{~d}, 1 \mathrm{H}, J=3.2 \mathrm{~Hz}), 5.10(\mathrm{dd}, 1 \mathrm{H}, J=4.4,8.4 \mathrm{~Hz}), 4.14(\mathrm{q}, 2 \mathrm{H}$, $J=7.1 \mathrm{~Hz}), 2.86(\mathrm{dd}, 1 \mathrm{H}, J=8.4,16.3 \mathrm{~Hz}), 2.78(\mathrm{dd}, 1 \mathrm{H}, J=4.4,16.3 \mathrm{~Hz}), 1.22(\mathrm{t}, 3 \mathrm{H}$, $J=7.1 \mathrm{~Hz})$.

1-(Benzofuran-2-yl)ethan-1-ol (2e): ${ }^{[2]}$ The agate beaker containing ten agate milling balls was filled with 1c $(80 \mathrm{mg}, 0.50 \mathrm{mmol}), \mathrm{NaBH}_{4}(189 \mathrm{mg}, 5.0 \mathrm{mmol})$ and choline chloride $(200 \mathrm{mg})$ and milling was performed for $1 \mathrm{~h}$. The reaction mixture was diluted with $\mathrm{CHCl}_{3}(10 \mathrm{~mL})$ and filtrated. The organic phase was washed with brine $(10 \mathrm{~mL})$, dried over $\mathrm{MgSO}_{4}$ and the solvent was removed under reduced pressure. 2 e $(58 \mathrm{mg}, 72 \%)$ was thus obtained as colourless solid. ${ }^{1} \mathrm{H}$ NMR $\left(300 \mathrm{MHz}, \mathrm{CDCl}_{3}\right): \delta=7.56-7.52(\mathrm{~m}, 1 \mathrm{H}), 7.46(\mathrm{~d}, 1 \mathrm{H}, J=7.2 \mathrm{~Hz}), 7.30-7.25(\mathrm{~m}, 1 \mathrm{H})$, 7.25-7.18 (m, 1H), $6.60(\mathrm{~s}, 1 \mathrm{H}), 5.07-4.96(\mathrm{~m}, 1 \mathrm{H}), 2.37(\mathrm{bs}, 1 \mathrm{H}), 1.64(\mathrm{~d}, 3 \mathrm{H}, J=6.6 \mathrm{~Hz})$.

6-Hydroxy-2-methyl-2H-pyran-3(6H)-one $\quad(\mathbf{3 b})::^{[3]}$ Quartz sand $(2.00 \mathrm{~g}), \quad \mathbf{2 b} \quad(210 \mu \mathrm{L}$, $2.00 \mathrm{mmol}$ ) and $\mathrm{mCPBA}(694 \mathrm{mg}, 4.00 \mathrm{mmol})$ were added together with ten agate milling balls in an agate beaker and milling was performed for $15 \mathrm{~min}$. The reaction mixture was diluted with water $(25 \mathrm{~mL})$ and filtered. The water was removed by lyophilization and $\mathbf{3 b}(182 \mathrm{mg}, 71 \%$ [dr 2:1]) was thus obtained as yellow solid. ${ }^{1} \mathrm{H}-\mathrm{NMR}\left(400 \mathrm{MHz}, \mathrm{CDCl}_{3}\right)$ : cis-isomer: $\delta=6.89$ (dd, $1 \mathrm{H}, J=3.4,10.3 \mathrm{~Hz}), 6.10(\mathrm{~d}, 1 \mathrm{H}, J=10.2 \mathrm{~Hz}), 5.63(\mathrm{~d}, 1 \mathrm{H}, J=3.4 \mathrm{~Hz}), 4.71(\mathrm{q}, 1 \mathrm{H}, J=6.8$ Hz), 1.39 (d, 3H, $J=6.8 \mathrm{~Hz}$ ); trans-isomer: $\delta=6.94(\mathrm{dd}, 1 \mathrm{H}, J=1.4,10.3 \mathrm{~Hz}), 6.15(\mathrm{dd}, 1 \mathrm{H}$, 
$J=1.6,10.3 \mathrm{~Hz}), 5.68(\mathrm{~d}, 1 \mathrm{H}, J=1.4 \mathrm{~Hz}), 4.22(\mathrm{qd}, 1 \mathrm{H}, J=1.2,6.7 \mathrm{~Hz}), 1.45(\mathrm{~d}, 3 \mathrm{H}, J=6.7$ $\mathrm{Hz})$.

Ethyl 2-(6-hydroxy-3-oxo-3,6-dihydro-2H-pyran-2-yl)acetate $\left.(3 \mathrm{c}):^{[4,}, 5\right]$ A solution of $2 \mathrm{c}$ $(257 \mathrm{mg}, 1.40 \mathrm{mmol})$ in $\mathrm{Et}_{2} \mathrm{O}(3 \mathrm{~mL})$ was mixed with quartz sand $(2.00 \mathrm{~g})$ and the solvent was removed under reduced pressure. The mixture was filled to an agate beaker equipped with ten agate milling balls, $m$ CPBA $(552 \mathrm{mg}, 3.20 \mathrm{mmol})$ was added and milling was performed for $30 \mathrm{~min}$. The mixture was diluted with water $(20 \mathrm{~mL})$, filtered and the water was removed by lyophilization. The crude product was purified by flash column chromatography (PE/EtOAc: 1/1) and 3c $\left(238 \mathrm{mg}, 84 \%\right.$ [dr 3:1]) was thus obtained as yellow-brown oil. ${ }^{1} \mathrm{H}-\mathrm{NMR}(400 \mathrm{MHz}$, $\left.\mathrm{CDCl}_{3}\right)$ : major diastereomer: $\delta=6.92(\mathrm{dd}, 1 \mathrm{H}, J=3.5,10.3 \mathrm{~Hz}), 6.14(\mathrm{~d}, 1 \mathrm{H}, J=10.3 \mathrm{~Hz}), 5.63$ $(\mathrm{d}, 1 \mathrm{H}, J=3.5 \mathrm{~Hz}), 5.02(\mathrm{dd}, 1 \mathrm{H}, J=3.9,7.6 \mathrm{~Hz}), 4.15(\mathrm{q}, 2 \mathrm{H}, J=7.1 \mathrm{~Hz}), 2.99(\mathrm{dd}, 1 \mathrm{H}, J=4.0$, $16.8 \mathrm{~Hz}), 2.74(\mathrm{dd}, 1 \mathrm{H}, J=7.6,16.7 \mathrm{~Hz}), 1.25(\mathrm{t}, 3 \mathrm{H}, J=7.2 \mathrm{~Hz}) ;$ minor diastereomer: $\delta=6.96$ (dd, $1 \mathrm{H}, J=1.6,10.3 \mathrm{~Hz}), 6.18(\mathrm{dd}, 1 \mathrm{H}, J=1.6,10.3 \mathrm{~Hz}), 5.73-5.70(\mathrm{~m}, 1 \mathrm{H}), 4.60-4.55(\mathrm{~m}, 1 \mathrm{H})$, $4.16(\mathrm{q}, 2 \mathrm{H}, J=7.1 \mathrm{~Hz}), 3.02(\mathrm{~d}, 1 \mathrm{H}, J=16.7 \mathrm{~Hz}), 2.82(\mathrm{~d}, 1 \mathrm{H}, J=7.9 \mathrm{~Hz}), 1.26(\mathrm{t}, 3 \mathrm{H}, J=7.1$ $\mathrm{Hz})$.

6-Hydroxy-6-(hydroxymethyl)-2H-pyran-3(6H)-one (3d): ${ }^{[6]}$ The agate beaker was equipped with ten agate milling balls, 2d $(41 \mathrm{mg}, 0.32 \mathrm{mmol})$, choline chloride $(40 \mathrm{mg})$ and $\mathrm{mCPBA}$ $(111 \mathrm{mg}, 0.64 \mathrm{mmol})$ and milling was performed for $60 \mathrm{~min}$. The reaction mixture was diluted with EtOAc $(20 \mathrm{~mL})$, filtered and the solvent was removed under reduced pressure. The crude product was dissolved in water $(20 \mathrm{~mL})$, filtered and the water was removed by lyophilization to obtain a 5:1 mixture of $\mathbf{3 d}(29 \mathrm{mg}, 63 \%)$ and the corresponding hemiacetal $\mathbf{5}$ as red brown oil. ${ }^{1} \mathrm{H}-\mathrm{NMR}\left(400 \mathrm{MHz}, \mathrm{CDCl}_{3}\right): 3 d: \delta=6.84(\mathrm{~d}, 1 \mathrm{H}, J=10.4 \mathrm{~Hz}), 6.19(\mathrm{~d}, 1 \mathrm{H}, J=10.4 \mathrm{~Hz}), 4.61$ $(\mathrm{d}, 1 \mathrm{H}, J=16.9 \mathrm{~Hz}), 4.16(\mathrm{~d}, 1 \mathrm{H}, J=16.9 \mathrm{~Hz}), 3.81(\mathrm{~d}, 1 \mathrm{H}, J=11.4 \mathrm{~Hz}), 3.64(\mathrm{~d}, 1 \mathrm{H}, J=11.5$ $\mathrm{Hz}) ; 5$ : $6.90(\mathrm{~d}, 1 \mathrm{H}, J=10.4 \mathrm{~Hz}), 6.18(\mathrm{~d}, 1 \mathrm{H}, J=10.4 \mathrm{~Hz}), 4.60(\mathrm{~d}, 1 \mathrm{H}, J=16.9 \mathrm{~Hz}), 4.46(\mathrm{~d}$, $1 \mathrm{H}, J=11.7 \mathrm{~Hz}), 4.19(\mathrm{~d}, 1 \mathrm{H}, J=16.9 \mathrm{~Hz}), 4.08(\mathrm{~d}, 1 \mathrm{H}, J=11.8 \mathrm{~Hz})$.

\section{References:}

[1] P. Srihari, Y. Sridhar, Eur. J. Org. Chem. 2011, 2011, 6690-97.

[2] C. Paizs, M. Toşa, C. Majdik, P. Moldovan, L. Novák, P. Kolonits, A. Marcovici, F.-D. Irimie, L. Poppe, Tetrahedron: Asymmetry 2003, 14, 1495-501.

[3] O. Achmatowicz Jr, P. Bukowski, B. Szechner, Z. Zwierzchowska, A. Zamojski, Tetrahedron 1971, 27, 1973-96.

[4] D. Noutsias, I. Alexopoulou, T. Montagnon, G. Vassilikogiannakis, Green Chem. 2012, 14, 601-04.

[5] M. Kusakabe, Y. Kitano, Y. Kobayashi, F. Sato, J. Org. Chem. 1989, 54, 2085-91.

[6] Y.-H. Kuo, K.-S. Shih, Heterocycles 1990, 31, 1941-49. 